\title{
图HAD
}

DOI: http://doi.org/10.22585/hospdomic.v4i4.118

\section{La atención en casa: El apoyo familiar en el control glicémico en pacientes con diabetes mellitus tipo 2}

\section{Home care: Family support in glycemic control in patients with type 2 diabetes mellitus}

Josefina Reynoso-Vázquez', Erik Hernández-Rivero², Mirel Martínez-Villamil2, Jennifer Lisaid Zamudio-López ${ }^{2}$, Iracema Islas-Vega ${ }^{3}$, Aldo Pelcastre-Neri ${ }^{3}$, Beatriz Garnica-Guerrero ${ }^{4}$, Jesús Carlos Ruvalcaba-Ledezma ${ }^{5}$

1. Universidad Autónoma del Estado de Hidalgo, Departamento de Farmacia y Salud Pública, Pachuca de Soto, México.

2. Universidad Autónoma del Estado de Hidalgo, Licenciatura de Farmacia, Maestría en Pachuca de Soto, México.

3. Universidad Autónoma del Estado de Hidalgo, Maestría en Salud Pública, Pachuca de Soto, México.

4. Universidad Autónoma de Durango, Licenciatura en Nutrición, Durango, México.

5. Universidad Autónoma del Estado de Hidalgo, Departamento de Medicina y Salud Pública, Maestría en Pachuca de Soto, México.

Correspondencia/Correspondence

Jesús Carlos Ruvalcaba Ledezma.

dcspicarlos@gmail.com

Circuito Ex Hacienda La Concepción S/N

Carretera Pachuca - Actopan, San Agustín

Tlaxiaca, Hidalgo.

01177117172000 Ext. 4301

Recibido/Received

16.08.2020

Aceptado/Accepted

04.10.2020
Conflicto de Intereses/Competing interest

No existió financiación.

Los autores y autoras declaran que no existe conflicło de intereses para la publicación del presente artículo.

CÓMO CITAR ESTE TRABAJO | HOW TO CITE THIS PAPER

Reynoso-Vázquez J, Hernández-Rivero E, Martínez-Villamil M, Zamudio-López J L, Islas-Vega I, PelcastreNeri A, et al. La atención en casa: El apoyo familiar en el control glicémico en pacientes con diabetes mellitus tipo 2. Hosp Domic. 2020;4(4): 199-207. 


\section{RESUMEN}

Introducción. La diabetes mellitus tipo 2 (DM2) es una enfermedad en la que el sujeto presenta alteraciones metabólicas de los carbohidratos, proteínas y grasas, de origen genético, por una deficiencia de la secreción de insulina o por la incapacidad para utilizarla, con grados variables de resistencia a ésta. Un individuo con diabetes debe de cambiar su estilo de vida, para lo cual es importante que reciba ayuda, generalmente de su familia.

Objetivo: Describir la trascendencia entre la atención en casa y/o apoyo familiar de la persona que vive con diabetes mellitus tipo 2 en lo que respecta al control glucémico.

Método: Estudio observacional, transversal en pacientes DM Tipo 2. Se aplicó 100 encuestas para la recolección de información que midió el apoyo familiar percibido por el paciente diabético.

Resultados: El promedio de edad de los participantes fue de 45 años, el $55 \%$ de la muestra correspondió al sexo masculino. El $57 \%$ de la población presenta niveles glucémicos superiores a $200 \mathrm{mg} / \mathrm{dl}$. 27\% presentan apoyo familiar y este es menor en las mujeres.

Conclusión: De acuerdo con los resultados obtenidos se concluye que el apoyo familiar es uno de los factores que influyen directamente con el cumplimiento del tratamiento farmacológico y no farmacológico.

Palabras clave: Servicios de Atención de Salud a Domicilio; Familia; Apoyo Social; Diabetes MeIlitus; Glucemia; Índice Glucémico; Terapéutica.

\section{ABSTRACT}

Introduction: Diabetes mellitus type 2 (DM2) is a disease in which the subject presents metabolic alterations of carbohydrates, proteins and fats, of genetic origin, due to a deficiency in insulin secretion or due to the inability to use it, with variable degrees of insulin resistance to it. An individual with diabetes should change their lifestyle, for which it is important that they receive help, usually from their family.

Objective: Describe the importance of home care and / or family support for the person living with type 2 diabetes mellitus in regard to glycemic control.

Method: Observational, cross-sectional study in DM Type 2 patients. 100 surveys were applied to collect information that measured the family support perceived by the diabetic patient.

Results: The average age of the participants was 45 years, $55 \%$ of the sample corresponded to the male sex. $57 \%$ of the population have glycemic levels higher than $200 \mathrm{mg} / \mathrm{dl}$. 27\% have family support and this is lower in women.

Conclusion: According to the results obtained, it is concluded that family support is one of the factors that directly influence the compliance of pharmacological and non-pharmacological treatment.

Keywords: Home Care Services; Family; Social Support; Diabetes Mellitus; Blood Glucose; Glycemic Index; Therapeutics. 


\section{INTRODUCCIÓN}

La diabetes mellitus (DM) es una enfermedad determinada genéticamente en la que el sujeto presenta alteraciones en el metabolismo de carbohidratos, de las proteínas y grasas, y una relativa o absoluta deficiencia de la secreción de insulina con grados variables de resistencia a ésta. Constituye un grave problema de salud pública. México está en la lista de los 10 países con mayor número de personas que viven con diabetes (1).

La Encuesta Nacional de Salud y Nutrición de Medio Camino 2016 exploró el estado de diversas enfermedades crónicas en México, entre ellas, la diabetes en la población mexicana mayor de 20 años de edad. Se encontró que la prevalencia de diabetes en el país pasó de 9.2\% en 2012 a 9.4\% en 2016, esto en base a un diagnóstico previo de la enfermedad. Entre esta población las mujeres reportan mayores valores de diabetes (10.3\%) que los hombres (8.4\%). Esta tendencia se observa tanto en localidades urbanas ( $10.5 \%$ en mujeres y $8.2 \%$ en hombres) como en rurales $(9.5 \%$ en mujeres, $8.9 \%$ en hombres). La mayor prevalencia de diabetes se observa entre los hombres de 60 a 69 años (27.7\%), y las mujeres de este mismo rango de edad (32.7\%) y de 70 a 79 años (29.8\%) (2).

En el Estado de Hidalgo se han registrado 3 mil 701 casos de diabetes tipo 2 (no insulinodependiente). Para el año 2018 se reportaron 9 mil 297 casos, de acuerdo con la Dirección General de Vigilancia Epidemiológica (3).

La prevalencia de DM2 entre 2010 y 2015 varió entre 0,8\% (Benín, África) y 37,3\% (Micronesia, Pacífico Occidental). Los países que notificaron menor prevalencia de DM2 (entre 0,8 y 2,2\%) durante los seis años analizados fueron Benín, Malí, Ruanda, Mongolia, Islandia, Burundi, Gambia, Uganda, Burkina Faso, Guinea, Guinea Bissau, Níger, Senegal y Sierra Leona, de los cuales 85,7\% pertenecían a África. Los países con mayor prevalencia de DM2 (entre 20,0 y 37,3\%) en el mismo periodo fueron Micronesia, Kiribati, Arabia Saudita, Baréin, Kuwait, Qatar, Vanuatu, Mauricio, Líbano y Palau, de los cuales 50,0\% pertenecían a Oriente Medio y Norte de África (4).

El aumento en la prevalencia de diabetes puede deberse al envejecimiento de la población, al incremento en la prevalencia de la obesidad relacionada con cambios en los estilos de vida (aumento en la densidad calórica de la dieta, reducción en la actividad física). Las causas que desencadenan la diabetes tipo 2 se desconocen en el 70-85\% de los pacientes; al parecer, influyen diversos factores como la herencia poligénica (en la que participa un número indeterminado de genes), junto con factores de riesgo que incluyen la obesidad, dislipidemia, hipertensión arterial, historia familiar de diabetes, dieta rica en carbohidratos, factores hormonales y una vida sedentaria (5).

Un individuo con diabetes se dirige casi siempre a su familia en busca de ayuda y consejo para solucionar sus problemas de salud; la respuesta que le brinden influirá positiva o negativamente sobre su conducta terapéutica y, por lo tanto, en el control de la diabetes (6).

La familia es sumamente importante en el contexto social, además de esto representa una base sólida para en enfrentamiento de diversos problemas, ya sea que se necesite un consejo por parte de un hermano o que se requiera el apoyo para realizar una acción, que tengamos duda de algún tema y debamos preguntar a cualquier miembro de la familia que este cerca. Al respecto la mayoría de pacientes obtienen información sobre su autocuidado de su propia familia, y con menor frecuencia recurren a fuentes de información como son el médico y la enfermera (7).

El término familia se considera como una entidad dentro de la sociedad en la cual todos los individuos tienen una función determinada o varias de ellas, también es llamado como núcleo familiar en el cual los individuos crecen y se desarrollan, además de que aprenden formas de convivencia, afrontamiento y expresión de sentimientos. De esta forma, cada uno de los integrantes de la familia tiene una concepción basta de conocimiento empírico que ha ido desarrollando a lo largo de la 
vida, sin embargo, no todos los individuos desarrollan las mismas capacidades, como por ejemplo de afrontamiento ante una enfermedad (8).

Su conformación está dada principalmente por parientes, o sea que por razones de sangre (consanguinidad), afinidad, adopción u otras razones más, hayan sido consideradas como miembros de ese núcleo (9).

El tipo y cantidad de ayuda que requiere el diabético dependerán del grado en que puede satisfacer las necesidades que se generan por el padecimiento Estas necesidades se agudizan conforme el padecimiento deteriora el funcionamiento del paciente, sobre todo cuando no se observa en forma estricta el tratamiento médico, apresurando los procesos degenerativos. Es entonces cuando la familia juega un papel decisivo en el control del paciente diabético (10-12).

\section{Objetivo general}

Describir la trascendencia entre la atención en casa y/o apoyo familiar de la persona que vive con diabetes mellitus tipo 2 en lo que respecta al control glucémico.

\section{MÉTODOS}

Se diseñó un estudio observacional y transversal en pacientes DM Tipo 2. Mediante la aplicación de una encuesta para la recolección de información que midió el apoyo familiar percibido por el paciente diabético, se realizó análisis descriptivo, se calcularon medidas de tendencia central y de dispersión para variables continuas y para variables cualitativas se obtuvieron razones y proporciones.

La encuesta estuvo conformada por 13 ítems los cuales evaluaron el apoyo tangible o instrumental y apoyo informacional que consta de tres áreas: 1). Conocimientos de la familia relacionados con las medidas de control y complicaciones de la DM2 para brindar apoyo informacional; 2). Actitudes familiares hacia el enfermo como apoyo emocional y 3). Medidas de control siendo el apoyo tangible.

El apoyo familiar es clasificado en categorías de excelente, bueno, regular y malo de acuerdo con la puntuación obtenida (Tabla 1). Para la calificación se utilizó la escala de Likert con tres alternativas de respuestas; frecuentemente, algunas veces, nunca.

Tabla 1: Categorías según el puntaje obtenido

\begin{tabular}{|l|l|}
\hline Categoría & Intervalo \\
\hline Excelente & $10-20$ \\
\hline Bueno & $21-30$ \\
\hline Regular & $31-40$ \\
\hline Malo & $41-50$ \\
\hline
\end{tabular}

Fuente: Directa, 2020 


\section{RESULTADOS}

Se incluyeron en el análisis a 100 personas con DM2. En la tabla 2 se presentan las características sociodemográficas de la población estudiada. La edad fluctuó entre 45 y 60 años, con una mediana de edad de 52 años. Se observó predominio del sexo masculino (55\%). Con respecto al estado civil, más de 78\% de los pacientes reportó estar casado o vivir en unión libre.

El nivel de escolaridad reportado con mayor frecuencia fue secundaria completa en un $81 \%$. El $40 \%$ de las personas evaluadas fueron amas de casa y el $60 \%$ comerciantes u obreros. El tratamiento farmacológico reportado con mayor frecuencia fue la utilización de antidiabéticos orales (ADO), como la glibenclamida, metformina y acarbosa.

Tabla 2: Características sociodemográficas de los pacientes con Diabetes Mellitus tipo 2

\begin{tabular}{|c|c|c|}
\hline Características & $N=100$ & $\%$ \\
\hline Edad (años) & 52 & $(45,60)$ \\
\hline $\begin{array}{l}\text { Sexo } \\
\text { Hombres } \\
\text { Mujeres }\end{array}$ & $\begin{array}{l}55 \\
45\end{array}$ & $\begin{array}{l}55 \% \\
45 \%\end{array}$ \\
\hline $\begin{array}{l}\text { Estado civil } \\
\text { Casado o unión libre } \\
\text { Soltero, divorciado, viudo }\end{array}$ & $\begin{array}{l}78 \\
22\end{array}$ & $\begin{array}{l}78 \% \\
22 \%\end{array}$ \\
\hline $\begin{array}{l}\text { Escolaridad } \\
\text { Primaria incompleta } \\
\text { Primaria y secundaria completa } \\
\text { Preparatoria o bachillerato universidad }\end{array}$ & $\begin{array}{l}10 \\
81 \\
9\end{array}$ & $\begin{array}{l}10 \% \\
81 \% \\
9 \%\end{array}$ \\
\hline $\begin{array}{l}\text { Ocupación } \\
\text { Amas de casa } \\
\text { No profesional } \\
\text { Profesional }\end{array}$ & $\begin{array}{l}40 \\
59 \\
1\end{array}$ & $\begin{array}{l}40 \% \\
59 \% \\
1 \%\end{array}$ \\
\hline Actualmente viven con familiares & 87 & $87 \%$ \\
\hline
\end{tabular}

Fuente: directa. Elaboración propia, 2020

Respecto al apoyo familiar, se valoró el apoyo material que percibe el paciente, la retroalimentación, la asistencia conductual. la interacción familiar y la conducta de guía- enseñanza. con respecto a la ayuda material se valoró la compra de medicamento, compra de alimentos de la dieta para diabético y pago de exámenes de laboratorio. se encontró que al 19\% siempre le compran el medicamento, al $35 \%$ por ciento siempre le compran sus alimentos y al $9 \%$ siempre le pagan los exámenes de laboratorio (Figura1). 
Figura 1: Apoyo familiar percibido por ayuda material (Fuente: directa, elaboración propia, 2020)

\section{Gráfico 1: Apoyo familiar percebido por ayuda material}

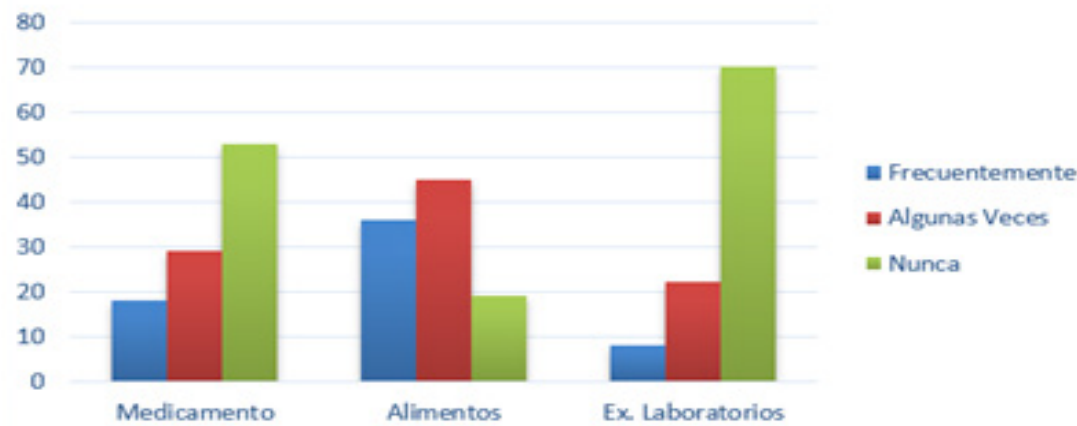

Referente a la retroalimentación se valoró el estímulo que el paciente recibe de su familiar para realizar ejercicio y si recibe felicitaciones cuando su peso y resultados de exámenes de laboratorio están normales, al respecto se obtuvo para el grupo de diabéticos en control que al 18\% nunca lo animan a realizar ejercicio, el $40 \%$ frecuentemente lo felicitan por su control de peso y que al $10 \%$ frecuentemente lo felicitan por mantener normales sus cifras de glucosa (Figura 2).

Figura 2: Apoyo familiar percibido por retroalimentación (Fuente: directa, elaboración propia, 2020)

\section{Gráfico 2: Apoyo familiar percibido por retrolimentación}

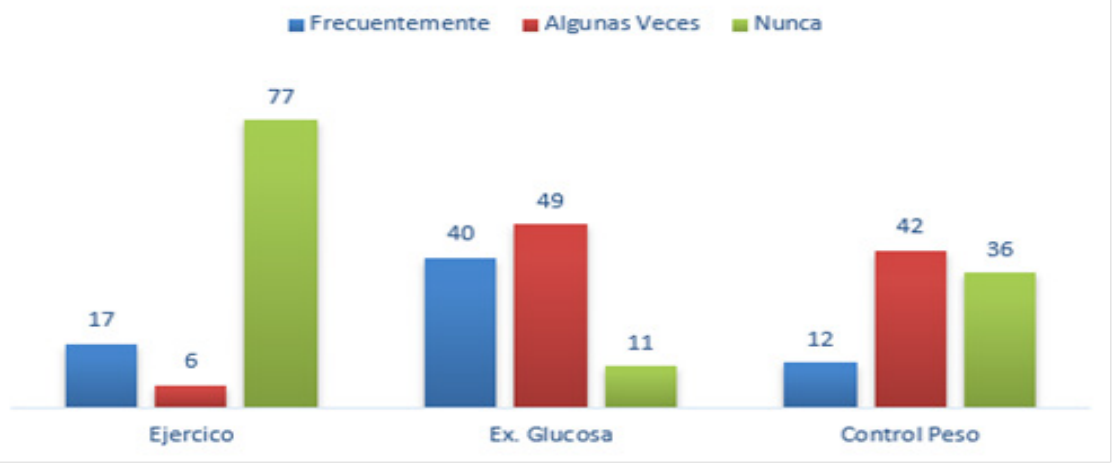

Referente a la interacción familiar., se manifestó por la frecuencia con que su familia ingiere los mismos alimentos que comprenden la dieta para diabético y la frecuencia con que le recuerdan la hora para que tome su medicamento, se encontró que en el grupo de diabéticos en control el $23 \%$ de los familiares ingieren frecuentemente los mismos alimentos de dieta para diabético y que al $40 \%$ de los pacientes su familiar le recuerda frecuentemente la hora para que tome sus medicamentos (Figura 3). 
Figura 3: Apoyo familiar percibido por interacción familiar (Fuente: directa, elaboración propia, 2020)

\section{Gráfico 3: Apoyo familiar percebido por interacción familiar}

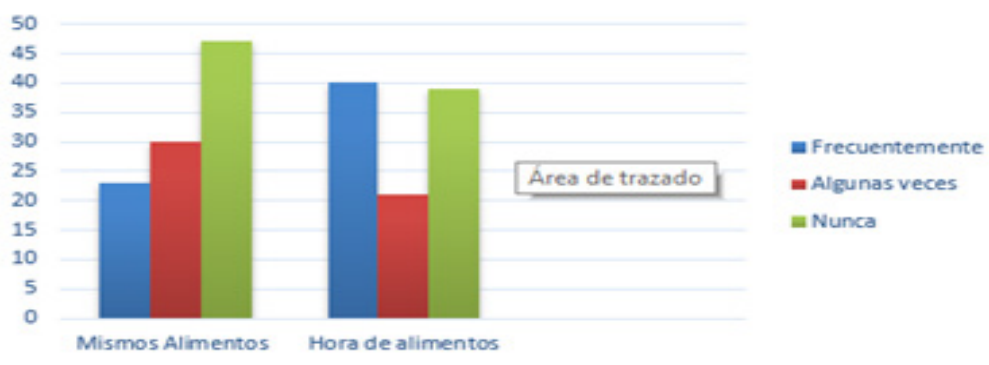

\section{DISCUSIÓN}

El control de la glucemia, más que el simple hecho de tomar un medicamento depende de un conjunto de conductas adoptadas frente a la enfermedad, en las que la familia interviene con su atención y/o apoyo para que la persona que vive con diabetes considere cotidiano la auto vigilancia y toma de sus medicamentos, realizar actividad física y adherirse a la dieta que en su alimentación está representada por alimentos con bajo índice glucémico.

Los resultados del presente estudio muestran que existe asociación entre el apoyo familiar percibido y el control glucémico en los pacientes diabéticos, dicha asociación resulta hasta este momento probable y lógica, lo que evoca a continuar con este tipo de estudios donde se busque probar de manera efectiva esta asociación.

Chávez-Zegarra realizaron una investigación en 142 pacientes que asistían a consulta externa de Endocrinología y determinaron que $47.2 \%$ tenía control glucémico, lo que no es similar a lo encontrado en este trabajo, en relación con el nivel del apoyo familiar, obtuvieron que 58.4\% tenía un nivel de apoyo familiar alto, $22.5 \%$ regular y $19 \%$ bajo, a diferencia del presente estudio en el que se determinó una menor frecuencia de apoyo familiar con 27\% (13).

En el trabajo de Merodio et al. se analizaron 113 pacientes adultos mayores de 60 años con diabetes, reportaron que $44.1 \%$ de los hombres recibía un buen apoyo familiar; el cual era menor en las mujeres, con $15.2 \%$, lo cual es similar con los resultados obtenidos en este estudio (14).

Dentro de las limitaciones de este trabajo se puede mencionar que solo se identificó la perspectiva del paciente. Sin embargo, hubiese sido conveniente realizar la encuesta tanto al paciente como al familiar de forma individual, para identificar la perspectiva desde ambas partes y evaluar su asociación con el control glucémico y tomar como parámetro de control la hemoglobina glucosada en lugar de glucosa en ayuno.

\section{CONCLUSIONES}

La diabetes mellitus tipo 2 es una de las enfermedades más comunes en la actualidad, sin embargo, sino se trata adecuadamente puede desencadenar serias complicaciones, afortunadamente hoy en día los tratamientos son muy eficaces, accesibles y seguros. 
A pesar de eso de acuerdo con los resultados obtenidos se tiene que el apoyo familiar es uno de los factores que influyen directamente con el cumplimiento del tratamiento farmacológico y no farmacológico. En esta investigación epidemiológica refleja datos negativos, en donde el apoyo familiar es bajo, ya que es poco frecuente que los familiares realicen actividades que benefician el estilo de vida de la persona que vive con diabetes, de aquí la trascendencia de contar con atención domiciliaria por parte de los familiares.

Un aspecto que es de importancia para tener una solución ante esta problemática es concientizar no sólo al paciente sino a los familiares sobre la importancia que tiene la atención y/o apoyo familiar en casa, así como de las consecuencias de no prestarle atención y seguimiento, apoyo cotidiano, ya que deben ser esfuerzos en conjunto para vivir con diabetes con control glucémico aceptable.

\section{BIBLIOGRAFÍA}

1. International Diabetes Federation (IDF). Atlas IDF 8 Edición - 2017 [página Web]. 2017 [accedido 2 feb 2020]. Disponible en: http://fmdiabetes.org/atlas-idf-2017/

2. Secretaría de Salud, Instituto Nacional de Salud Pública. Encuesta Nacional de Salud y Nutrición de Medio Camino 2016 (ENSANUT MC 2016) [monografía en Internet]. México: Instituto Nacional de Salud Pública; 2016 [accedido 12 abr 2020]. Disponible en: http://fmdiabetes.org/ wp-content/uploads/2017/04/ENSANUT2016-mc.pdf

3. Dirección General de Epidemiología (DGE). Anuario de morbilidad 1984-2018 [página Web]. México: Gobierno de México; 2020 [accedido 12 abr 2020]. Disponible en: https://epidemiologia.salud.gob.mx/anuario/html/anuarios.html

4. Mendoza Romo MA, Padrón Salas A, Cossío Torres PE, Soria Orozco M. Prevalencia mundial de la diabetes mellitus tipo 2 y su relación con el índice de desarrollo humano. Rev Panam Salud Publica. 2017;41:e103. DOI: 10.26633/RPSP.2017.103

5. Cervantes-Villagrana RD, Presno-Bernal JM. Fisiopatología de la diabetes y los mecanismos de muerte de las células $\beta$ pancreáticas. Rev Endocrinol Nutr. 2013;21(3):98-106.

6. Ávila-Jiménez L, Cerón D, Ramos-Hernández RI, Velázquez L. Asociación del control glicémico con el apoyo familiar y el nivel de conocimientos en pacientes con diabetes tipo 2. Rev Med Chile. 2013;141(2):173-80.

7. Chapman Sánchez M, García Almaguer R, Caballero González G, Peneque Caballero Y, Sablón Mariño A. Efectividad de intervención educativa en el conocimiento del paciente diabético sobre autocuidados. Rev Cubana Enfermer. 2016;32(1):49-59.

8. García Escalante MG, Pinto Escalante D, Valadez González N. La Diabetes, mi Familia y Yo [monografía en Internet]. Mérida. México: CONACYT; 2014 [accedido 13 abr 2020]. Disponible en: https://bit.ly/3i6KQ8g

9. Asociación Mexicana de Diabetes (AMD). Familia y Diabetes [página Web]. México: AMD; [accedido 13 abr 2020]. Disponible en https://www.amdiabetes.org/

10. Valadez-Figueroa I de la A, Aldrete-Rodríguez MG, Alfaro-Alfaro N. Influencia de la familia en el control metabólico del paciente diabético tipo II. Salud Pública Mex.1993;35(5): 464-70. 
11. Rivera Vázquez P, Hernández Villanueva C, Carbajal Mata FE, Maldonado Guzmán G. Funcionalidad Familiar y Control Glicemico en Adultos Diabeticos Tipo2 en una Comunidad Rural de Tamaulias, México. Rev Car Cienc. Sos. 2016.

12. Alulima Salazar SM. La familia como factor desencadenante de descompensación en la salud integral y la adherencia al tratamiento de los pacientes con diabetes mellitus tipo II que asisten al servicio de clínica del hospital general Isidro Ayora de la ciudad de Loja, periodo. 2015-2016. Loja: Universidad Nacional de Loja; 2016

13. Chávez Zegarra GS. Relación entre el nivel de apoyo familiar en el cuidado y la calidad de vida de los pacientes con diabetes mellitus tipo 2, atendidos en la consulta externa del Hospital Nacional Guillermo Almenara Irigoyen, Lima, Perú. Rev Cient Cienc Salud. 2013;6(2):7-14.

14. Merodio Pérez Z, Rivas Acuña V, Martínez Serrano A. Percepción del apoyo familiar y dificultades relacionadas con la diabetes en el adulto mayor. Horizonte Sanitario. 2015;14(1):14-20. 